\title{
Sur les traversées
}

\author{
Entretien avec Sylvie Kandé
}

2019. «Sur les traversées. Entretien avec Sylvie Kandé », Multitudes, n76, 3, p. 188193

\section{Propos recueillis par Elara Bertho}

Sylvie Kandé est une poétesse qui navigue entre l'Afrique de l'Ouest, l'Europe et le continent américain. Ce dialogue tricontinental, reliant plusieurs mers et plusieurs océans, est au cour de sa pratique poétique. Lagons, lagunes. Tableau de mémoire (2000) est une série de tableaux juxtaposant des références à l'empire du Mali, Charles Baudelaire, Victor Hugo, tout autant que des martyrs de Saint Domingue. Ce tressage des influences se retrouve également dans son dernier recueil, Gestuaire (2016), où chaque poème constitue la mémoire d'un "geste» : les champs de coton, le créole, l'archaïsme français, l'hermétisme parfois cohabitent dans cette suite d'éclats de voix finement ciselés. C'est singulièrement La quête infinie de l'autre rive. Épopée en trois chants (2011) qui permet d'éclairer la nouvelle "sans histoire/s " qui précède cet entretien: dans ces trois chants, Sylvie Kandé propose trois traversées de l'Afrique de l'Ouest vers l'inconnu, de l'autre côté de la rive, superposant le $X I I I^{e}$ siècle malien et l'extrême contemporain.

Elara Bertho : la vie des « sans histoire ", c'est s'attacher à décrire des traces, des bribes de souvenirs, une "tache sur une feuille" pour reprendre l'exergue de Pentti Holappa, ou un simple nom. C'est à cette entreprise que vous vous attachez dans La quête infinie de l'autre rive (2011) qui décrit dans son chant III l'épopée des migrants, anonymisés sur nos écrans de télévision, qui luttent pour atteindre l'autre rive. Dans « sans histoire/s ", vous renouvelez cette pratique du récit des sans voix pour décrire le quotidien de Mori, étranger à New York qui a parcouru de nombreuses rives déjà, entre l'Afrique, Paris et New York, et qui s'évertue, surtout, à "ne pas faire d'histoire ". Quelle est pour vous l'importance, et peut-être l'urgence, de dire ces histoires de ceux qui désirent ne pas en avoir ?

Sylvie Kandé : Tenter de témoigner pour les migrants qui, en dépit de leur démunition relative ou absolue, sont partout aujourd'hui la cible de persécutions politiques et de discours imprécatoires, est, depuis longtemps, un axe majeur de mon travail. En témoigne Lagon, lagunes. Tableau de mémoire avec, notamment, le passage qui s'ouvre sur le vers : «Ils sont venus à l'aube comme à l'accoutumée... ${ }^{1}{ }^{1}$. Dans La quête infinie de l'autre rive, j'accompagnais en mots ceux et celles qui migrent par bateau, au Moyen-Âge comme aujourd'hui, persuadée qu'ils représentent la part la plus exigeante, la plus novatrice de notre humanité, celle qui "se refuse à croire qu'il n'y a rien au-delà de l'horizon ». Avec "sans histoire/s », je demande que l'on "considère » les histoires individuelles des migrants, en

\footnotetext{
${ }^{1}$ Sylvie Kandé, Lagon, lagunes. Tableau de mémoire. Gallimard, Continents noirs, 2000, p. 39-41.
} 
particulier de ceux qui, avant 2016, faisaient figure de migrants « sans histoires » et n'étaient pas inquiétés. La vie brisée de Mori est emblématique de l'acharnement avec lequel le régime sacrifie désormais ces hommes et ces femmes à une politique du panem et circenses. J'utilise ici «considérer » au sens où Marielle Macé l'a déployé : "C'est un mot, écrit-elle, de la perception et de la justice, de l'attention et du droit. Il désigne cette disposition où se conjugue le regard [...] et l'égard, le scrupule, l'accueil sérieux de ce que l'on doit faire effort pour garder sous les yeux. Devant des événements aussi violents que la 'crise des migrants', il est plus commun, plus immédiat, de se laisser sidérer que de considérer. $»^{2} \mathrm{~J}^{\prime}$ 'ai voulu, bien qu'il m'en coûte de le faire, "garder sous les yeux » et prendre aux mots, sérieusement, les violences que tant de Mori ont subies au cours des dernières années : arrestations à domicile ou sur leur lieu de travail, séjours en prison voire en réclusion solitaire, expulsions et retoursnaufrages au pays natal ${ }^{3}$.

Comme mes textes littéraires précédents, "sans histoire/s» a surgi au point de tangence entre thème et mot : savoir qu'on avait pris «Mori» m'a terrassée ; pour le dire avec Claude Mouchard, c'était « COMME UN COUP À (ou un agrippement sur) L'ÉPAULE,/ me forçant à me retourner sur ce qui était en train d'avoir lieu, dans mon dos, ... ${ }^{4}$. M'est soudain revenue l'image de cette voisine qui vivait seule dans le même immeuble que ma grand-mère. Des années de larmes avaient creusé des rigoles sur son visage qu'illuminait malgré tout un regard à la Modigliani. Chaque fois qu'en descendant l'escalier, nous approchions du pas de sa porte, ma grand-mère se retournait vers moi et posait un doigt sur ses lèvres. Car ce palier était le site sacré de la douleur inconsolable d'une mère : un jour, on était venu prendre son fils dont on lui avait renvoyé par colis, au bout d'un certain temps, les quelques possessions. Il n'était alors resté à cette femme que ses yeux bleus, immenses, pour pleurer.

Qu'il y ait mort d'hommes ou mort sociale d'individus, il importe de parler de ceux qui sont pris, innocents de tout crime mais rangés sans autre "considération » dans une catégorie légale ou identitaire d'indésirables, puis broyés. Faire des taches sur une feuille en leur nom, c'est redonner à ces exclus droit de cité sur la page, c'est offrir du papier à défaut de papiers. C'est faire en sorte qu'ils n'aient pas vécu en vain, pour en revenir au vers du poète Pentti Holappa; c'est aussi se préoccuper de re-nom puisque personne n'est l'égal d'une injuste réputation, selon la formule du penseur soufi Tierno Bokar.

E.B. : Le récit est éclaté par des bribes de voix autres qui viennent perturber la linéarité du texte et qui sont signalées par des changements typographiques. Parfois, il s'agit d'un narrateur qui apostrophe son propre récit, parfois d'une voix de femme peut-être qui se souvient de Mori : le récit se doit-il d'être choral pour se remémorer ce qui n'a été qu'un fait divers dans cette grande métropole américaine ?

S.K. : Qui a le droit de raconter l'histoire? Peut-on manipuler le drame des autres à des fins esthétiques ? Voilà en art de véritables motifs d'angoisse. En manière de parade à l'inauthenticité, à la condescendance bienveillante qui pourraient entacher la représentation des marginaux, mis au silence par le pouvoir ou décidés au mutisme par mesure de prudence, on multipliera les voix qui captent un peu de leur vérité. Dans mon récit, plusieurs interventions, dont celle d'un témoin «objectif » et celle de Mori lui-même (en italiques), tentent de remettre le récit sur les rails ou de le faire dérailler, c'est selon, défient les interprétations du narrateur/de la narratrice, ou surenchérissent : ce chœur vient en tous cas souligner la subjectivité du point de vue omniscient et autorisé. En défaisant la dichotomie

\footnotetext{
${ }^{2}$ Marielle Macé, Sidérer, considérer. Migrants en France, 2017. Verdier, 2017, p. 26.

${ }^{3} \mathrm{cf}$ https://www.metro.us/news/local-news/new-york/icewatch-ice-raids-map

${ }^{4}$ Claude Mouchard, Papiers! Pamphlet-poème. Editions Laurence Teper, 2007, p. 44.
} 
narrateur/sujet, on s'ouvre aussi à un mode de connaissance autre, basé sur la réciprocité. Glissant a évoqué cette possibilité : "Considérer le malheur des peuples. Non pas seulement par souci moral, mais parce que ce malheur, toujours offusqué ou oblitéré, entre pour une grande part dans notre connaissance du monde et de nous-mêmes $»^{5}$.

Je me connais donc mieux, je connais mieux mes «multiplicités» (un concept deleuzien qui spécialement m'interpelle) de connaître Mori, personnage autour duquel se nouent histoires, anecdotes, informations et racontars glanés de-ci de-là : «... je chante ce que j'ignorais que nous savions, moi, moi et moi...». Je me suis donc souvenue qu'une démunition extrême, telle qu'il en existe au milieu de l'abondance dans des villes comme New York, combinée à l'impression de ne pas pouvoir être «joueur » dans un monde où le coût de toute chose, jusqu'à celui du temps, est strictement calculé et à l'étrange familiarité des paysages urbains quand l'hiver se fait âpre, conduisent aisément au désespoir. En de telles circonstances, on pourrait bien, sous couvert de traversée spirituelle ${ }^{6}$, se jeter dans les eaux glacées du Hudson, comme Mori envisageait sans doute de le faire. Mais parfois, pour ceux qui se noient, il y a un inconnu dont la main tendue, ou placée «près du cœur où est la bonté », dit qu'il a mal à autrui. Et cette main « retouche» heureusement l'issue fatale d'une existence désespérée. "Toucher au cœur», observe William Berthomière dans un essai sur les images de presse évoquant les mains tendues en direction des réfugiés, " nous renvoie à l'idée toute derridienne d'hospitalité inconditionnelle ${ }^{7}$. C'est effectivement du devoir d'hospitalité à l'égard de ceux qui traversent tant d'épreuves dont il est question dans mon récit.

E.B. : Vous faites à de nombreuses reprises référence à Tierno Bokar, Le Sage de Bandiagara, d'Amadou Hampâté Bâ (1957). On y voit par exemple Tierno recoudre un nid tombé à terre par une bourrasque et accueillir un oisillon hirondelle parmi ses disciples. Ce message de tolérance, d'accueil de la diversité et de soin apporté à l'autre irrigue votre écriture également. Comment lisez-vous cet enseignement soufi ?

S.K. : Mon récit est linéaire sans l'être puisqu'après une exposition qui place l'histoire dans un moment politique précis, il suit Mori depuis le matin où il rentre à son domicile après une course de nuit jusqu'à son retour quelque part en Afrique où il vivote désormais, en «se débrouillant », miné par son « échec » — une manière bien imparfaite de dénouement. Cette linéarité est perturbée par trois moments de crise qui ne se suivent pas chronologiquement (la tentative de suicide par noyade du personnage, son arrestation à domicile, sa crise psychotique en réclusion solitaire suivie d'une seconde tentative de suicide). À l'imitation des tours et détours d'une réminiscence, l'histoire est tissée de détails grossis, de récits dans le récit (l'apologue de l'oisillon), d'un art poétique, de vers, d'interventions de personnages nonidentifiés, de flash-back et d'anticipation : c'est ainsi qu'on apprend le retour de Mori, sain et sauf, dans son appartement avant de savoir qui l'a sauvé de la noyade ; on connaît son expulsion avant d'avoir les détails de son arrestation. Un peu comme si le narrateur/la narratrice, résumant un épisode parfois trop rapidement au goût de l'auditoire, était ensuite sommé/e d'expliquer « comment ça s'est passé ».

\footnotetext{
${ }^{5}$ Édouard Glissant, Traité du Tout-Monde. Paris: Gallimard/nrf, 1997, p. 214

${ }^{6}$ Louis Brenner, West African Soufi. The Religious Heritage \& Spiritual Search of Cerno Bokae Saalif Taal. U. of California Press, 1984, p. 176. Dans la parabole du canot, Tierno Bokar suggère à qui veut traverser une rivière mystique de charger son âme de façon à équilibrer questions spirituelles et préoccupations matérielles.

${ }^{7}$ William Berthomiere « Touchant-touché. Mécanique politique de la main tendue. » Science And Video, MMSH (Aix-en-Provence), 2018, Réfugiés en images/Images de réfugiés, dirigé par Fabienne Le Houérou, http://scienceandvideo.mmsh.univ-aix.fr/numeros/7/Pages/03.aspx
} 
J'ai écrit « sans histoire/s » sur le mode de la broderie, avec des motifs qui se révèlent au moyen de mots-couleurs : " manières », « désarroi » ou " parfum » (le parfum que Mori associait à Paris/qu'il aurait souhaité qu'on lui rapporte de voyage/cette " part du voyage » qu'on n'aura pas eu le temps de lui donner et qui, comme lui, s'est évaporé) sont de tels mots qui courent dans tout le texte et le colorent à leur façon. Incidemment, Tierno Bokar — dont on gagnerait à mieux connaître la pensée aujourd'hui qu'abondent les amalgames entre Islam et terrorisme - était tailleur-brodeur avant que d'accepter sa vocation de guide spirituel soufi $^{8}$. En s'invitant dans mon récit, qu'il traverse de sa présence lumineuse, il lui a donné, je crois, plus d'épaisseur.

C'est à la vue des moineaux que Mori se remémore une histoire qu'on lui avait racontée au sujet de Tierno Bokar. Un beau jour, ce dernier avait interrompu, dit-on, la leçon qu'il donnait pour porter secours à l'oisillon tombé du nid sous les yeux indifférents de ses disciples, assis dans la cour de sa maison. Ce faisant, le maître donnait une leçon plus précieuse encore : l'hospitalité doit s'adresser à tous, mais se porter d'abord vers les plus vulnérables ; à rebours de l'indifférence meurtrière, elle garantit notre humanité. Dans un geste d'infinie tendresse, Maître Bokar était même allé jusqu'à recoudre le nid d'hirondelle et y replacer le poussin. Avec davantage de délicatesse encore, il l'avait appelé " fils d'autrui », brouillant par là la frontière entre espèces et catégories qui est censée nous obliger différemment devant la souffrance des uns et des autres. Tout " enfant » est quelqu'un de précieux pour sa mère, nous enseigne-t-il donc, et tout hôte de ma maison est sacré, sans contrat de réciprocité.

Bien plus, son amour avait extrait ce poussin-là de la catégorie oiseau où il était indiscernable, fait fusionner ses multiplicités propres avec les siennes, de sorte que l'homme de Dieu était devenu le parent de l'oiselet, celui qui le protège : "Que veut dire aimer quelqu'un? » écrit Deleuze, " Toujours le saisir dans une masse, l'extraire d'un groupe, même restreint, auquel il participe, ne serait-ce que par sa famille ou par autre chose; et puis chercher ses propres meutes, les multiplicités qu'il enferme en lui, et qui sont peut-être d'une tout autre nature. Les joindre aux miennes, les faire pénétrer dans les miennes, et pénétrer les siennes $\iota^{9}$. De fait, le poussin d'hirondelle de cet apologue est probablement ce/lui qui nous parle le mieux de l'essor et du chant du maître soufi.

Le concept du «devenir-animal » deleuzien permettrait sans doute de rendre compte des traversées et interventions de l'animal dans mon récit. Mori ne ressemble pas à un oiseau, mais la bande de moineaux devant son immeuble, inquiète du chat qui rôde dans le petitmatin, l'avertit de son sort prochain. Dans l'enfer de sa cellule, un échassier le surveille et des chauves-souris s'échappent de sa tête qu'il frappe contre les murs pour tenter d'écraser leur vol noir. Et même si la lecture du roman d'Upton Sinclair, The Jungle (1906) et de l'essai de Timothy Pachirat, Every Twelve Seconds (2011) m'ont éclairée sur les parallèles entre l'exploitation animale et celle des travailleurs des abattoirs — où figure d'ailleurs un large pourcentage de migrants - , c'est plutôt le concept d' « animal-bordure » (celui qui représente et dissimule la masse de ses semblables) qu'on reconnaît dans cette vache que l'ami de chambrée de Mori se refuse à égorger. On imagine l'homme sortir en pleurs de l'abattoir; et son triomphe éthique (il n'a pas tué le « fils d'autrui ») le prive de ses modestes revenus - lui met en quelque sorte le couteau sous la gorge.

\section{E.B. : Mori est passeur des frontières, dans le passé il a voyagé jusqu'à Paris, et dans le temps du récit également, il passe les contrôles autoroutiers et franchit de}

\footnotetext{
${ }^{8}$ Voir les travaux d'Amadou Hampâté Bâ, de Théodore Monod et de Louis Brenner à son sujet.

${ }^{9}$ Gilles Deleuze et Félix Guattarri, Mille Plateaux. Capitalisme et schizophrénie, Éditions de Minuit, 1980, p 49.
} 
nombreux seuils. Cette traversée permanente se traduit dans votre écriture par une prise en charge de nombreuses autres langues par le français, qui se voit ainsi émaillé d'anglais, d'espagnol... Est-ce pour vous une réponse par la langue à certaines revendications identitaires qui se crispent aujourd'hui, pour montrer au contraire à quel point toutes les langues sont traversées sans cesse les unes par les autres ?

S.K. : En dépit de la célébration de l'entre-deux, de la création de l'espace Schengen, de la redéfinition de la migration comme droit de l'homme $^{10}$, le concept de frontière continue de modeler nos imaginaires et nos modes de vie. Pour s'en convaincre, il suffit d'examiner la structure d'un immeuble, ce «cube-à-cohabiter», divisé en appartements, chacun d'eux divisé à son tour en pièces délimitées par des portes censées protéger l'espace privé de son occupant : à chaque étape sont prévus des mécanismes de contrôle des entrées ou des sorties - clés, interphones, caméras, assistants personnels intelligents, etc. Pour qui immigre en situation précaire, rejouer ce premier passage de frontière dans toutes ses déclinaisons (péage, domicile, administration, écoles, etc.) ne peut que traumatiser.

Le Mori de mon récit me rappelle les pacotilleuses décrites par Glissant dans son roman Tout-Monde. Elles vont d'un lieu à l'autre, " reliant la vie à la vie », charriant une marchandise qu'elles achètent ici pour la revendre là-bas, mais aussi «l'air et les commérages, le manger comme les préjugés, le beau soleil et les cyclones. " À l'aise dans le voyage, la traversée et la rencontre, elles sont les figures par excellence de la Relation, auxquelles l'écrivain se compare : «Disons, ce sera pour me vanter, je suis le pacotilleur de toutes ces histoires rassemblées $»^{11}$.

Mori, qui cultive à la fois dandysme et discrétion, est cosmopolite comme les pacotilleuses : s'il n'a pas leur bagout, s'il se fait un devoir d'être aussi «prose» que possible, il partage avec elles cette aisance dans la relation et cette faculté d'adaptation à la diversité du monde. Son plus grand bonheur, c'est d'employer l'expression juste au bon moment, dans l'esprit et la musique de la langue de l'autre. Sa pacotille, son trésor, ce sont les mots qu'il transporte, cajole et dont il renouvelle le sens à l'occasion.

À charge pour moi de recréer dans ma langue d'écriture, le français, l'univers linguistique de ce citoyen du monde, compétent en trois langues internationales au moins, outre sa ou ses langue/s maternelle/s, sans avoir appris à en lire aucune. J'ai voulu montrer que Mori, passeur de cultures, traducteur et fin sémiologue - la ville qu'il sillonne de jour comme de nuit n'a plus de secrets pour lui - vivait déjà ce Tout-Monde, cette Créolité où l'on pense «en présence de toutes les langues du monde », où le purisme, notamment linguistique, n'a pas de place. Il se trouve pourtant expulsé de ce lieu tiers, de ce sanctuaire qu'est New York, et ce, pour ne pas avoir tenu compte du rejet de sa demande d'asile. Ce processus brutal est aussi affaire de portes: des agents des services de contrôle de l'immigration clandestine pénètrent par ruse dans l'appartement du Bronx où il vit avec d'autres familles, l'arrachent de la chambre qu'il partageait avec deux autres célibataires ; on l'enferme en centre de détention puis en réclusion solitaire, avant de le refouler à la frontière où il est laissé-pour-compte.

Toute vie est une vie, comme l'énonce la Charte du Manden ${ }^{12}$. Le départ de cet ami des enfants, des mots et des langues, humble et «stylé », décent et déjanté, riche aussi de sa part d'opacité, appauvrit les parages dont il était l'hôte.

\footnotetext{
${ }^{10}$ Voir les travaux de Catherine Wihtol de Wenden et de Michel Agier sur cette question.

${ }^{11}$ Édouard Glissant, Tout-Monde. Gallimard/nrf, 1993, 460-467.

${ }^{12}$ La Charte du Manden (Charte de Kurukan Fuga) établit les relations entre individus dans l'empire de Soundiata Keita, qui régna sur l'empire du Mali au XIII ${ }^{\mathrm{e}}$ siècle. Il existe plusieurs versions de la Charte. Voir à ce sujet Celhto (Centre d'Études Linguistiques et Historiques par Tradition Orale), La Charte de Kurukan Fuga: aux sources d'une pensée politique en Afrique, Conakry, S.A.E.C./L'Harmattan, 2008. Voir le
} 
commentaire qu'en livre Souleymane Bachir Diagne dans son dialogue avec Jean-Loup Amselle, En quête d'Afrique(s), Universalisme et pensée décoloniale, Paris, Albin Michel, 2019, p. 261-267. 